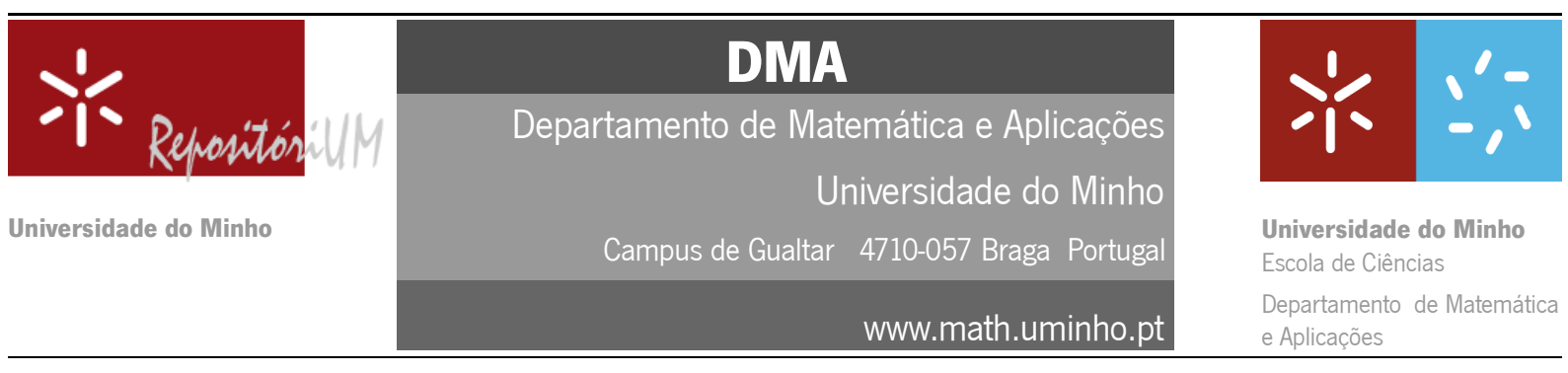

\title{
On Special Functions in the context of Clifford Analysis
}

\author{
H.R. Malonek ${ }^{a}$ \\ M.I. Falcão ${ }^{b}$
}

${ }^{a}$ Departamento de Matemática, Universidade de Aveiro, Portugal

${ }^{b}$ Departamento de Matemática e Aplicações, Universidade do Minho, Portugal

\section{Information}

Keywords:

Special Functions, Clifford Analysis, Appell sequences, combinatorial identities.
Original publication:

AIP Conference Proceedings, 2010, 1281, 1492-1495

DOI: $10.1063 / 1.3498054$

link.aip.org/link/?APCPCS/1281/1492/1

\begin{abstract}
Considering the foundation of Quaternionic Analysis by R. Fueter and his collaborators in the beginning of the 1930 s as starting point of Clifford Analysis, we can look back to 80 years of work in this field. However the interest in multivariate analysis using Clifford algebras only started to grow significantly in the 70 s. Since then a great amount of papers on Clifford Analysis referring different classes of Special Functions have appeared. This situation may have been triggered by a more systematic treatment of monogenic functions by their multiple series development derived from Gegenbauer or associated Legendre polynomials (and not only by their integral representation). Also approaches to Special Functions by means of algebraic methods, either Lie algebras or through Lie groups and symmetric spaces gained by that time importance and influenced their treatment in Clifford Analysis. In our talk we will rely on the generalization of the classical approach to Special Functions through differential equations with respect to the hypercomplex derivative, which is a more recently developed tool in Clifford Analysis. In this context special attention will be payed to the role of Special Functions as intermediator between continuous and discrete mathematics. This corresponds to a more recent trend in combinatorics, since it has been revealed that many algebraic structures have hidden combinatorial underpinnings.
\end{abstract}

The revival of Special Functions in the era of Scientific Computing is not surprising. If we have in mind that Special Functions are omnipresent in the theoretical background of any differential, integral or functional equation, and that the use of symbolic algebraic calculations simplifies the detection of interesting unknown properties, then we can understand that the amount of contributions on Special Functions and related subjects is again increasing. From this general point of view it also seems natural to ask for the role and the application of Special Functions in Clifford Analysis.

Since the theory of monogenic [2] or (Clifford holomorphic functions [6]) has its origin in complex function theory, one can expect that almost all properties of Special Function theory should have their counterpart. A 
first superficial look may confirm this standpoint, but two arguments exist that are in our opinion essential for a different judgement about the usefulness of studies on Special Functions in Clifford Analysis. First, it seems that the possible contribution to a different way of dealing with functions in several real variables could enrich the multidimensional theory of Special Functions. Second, the use of the non-commutative Clifford Algebra promises results which cannot be obtained in the usual multidimensional commutative setting.

In the beginning of Quaternionic Analysis the applications to Mathematical Physics were not in the center of attention. Maybe this explains why one cannot find any remark on generalized exponential function in the work of R. Fueter [4]. Nevertheless, in the 70s the interest in Clifford algebras grew again and papers referring to different classes of Special Functions appeared. One of the reasons was that the main way of simulating a general Weierstrass-like approach to monogenic functions by the development of the generalized Cauchy kernel by convergent multiple power series relied (see f.g. [2], [6]) directly on the use of Gegenbauer polynomials for obtaining a complete basis of polynomials for each homogeneous degree. Another reason was the fact that the set of monogenic functions is not closed with respect to multiplication and therefore the algebraic manipulations, for instances in transform analysis (see [2]), can be very complicated. It is often an easier task to use another complete basis of polynomials depending on the considered problem. Naturally this led to the use of other than Gegenbauer polynomials and the search of suitable polynomial bases is one of the main tasks in Clifford Analysis. But because an important general approach to the determination of such polynomial sets consists in using a suitable generating function, for example, of hypergeometric type, the appearance of Special Functions in Clifford Analysis is very natural.

However usually a coin has not only a bad side. If we combine, for example, the knowledge of different polynomial bases systems with the problem of establishing and proving new combinatorial identities by bijective methods, then it will be possible to take profit from the sometimes cumbersome search for the best suitable bases systems. As far as we know, enumerative combinatorics did not deal until now with the corresponding function classes arising in Clifford Analysis.

As starting point for the concrete explanation of the described principal ideas we choose an orthonormal base $\left\{e_{1}, e_{2}, \cdots, e_{n}\right\}$ of the Euclidean vector space $\mathbb{R}^{n}$ equipped with a product according to the multiplication rules $e_{k} e_{l}+e_{l} e_{k}=-2 \delta_{k l}, k, l=1, \cdots, n$, where $\delta_{k l}$ is the Kronecker symbol. This non-commutative product generates the $2^{n}$-dimensional Clifford algebra $C l_{0, n}$ over $\mathbb{R}$ and the set $\left\{e_{A}: A \subseteq\{1, \cdots, n\}\right\}$ with $e_{A}=e_{h_{1}} e_{h_{2}} \cdots e_{h_{r}}, 1 \leq h_{1} \leq \cdots \leq h_{r} \leq n, e_{\emptyset}=e_{0}=1$, forms a base of $C l_{0, n}$. The real vector space $\mathbb{R}^{n+1}$ will be embedded in $C l_{0, n}$ by identifying the element $\left(x_{0}, x_{1}, \cdots, x_{n}\right) \in \mathbb{R}^{n+1}$ with the para-vector $x=x_{0}+\underline{x}$, where $\underline{x}=e_{1} x_{1}+\cdots+e_{n} x_{n}$. Like in the complex case, the conjugate of $x$ is $\bar{x}=x_{0}-\underline{x}$ and the norm $|\bar{x}|$ of $x$ is defined by $|x|^{2}=x \bar{x}=\bar{x} x=x_{0}^{2}+x_{1}^{2}+\cdots+x_{n}^{2}$. Denote by $\omega(x)=\frac{x}{|\underline{x}|} \in S^{n}$, where $S^{n}$ is the unit sphere in $\mathbb{R}^{n}$.

In what follows we consider $C l_{0, n}$-valued functions defined in some open subset $\Omega \subset \mathbb{R}^{n+1}$, i.e., functions of the form $f(z)=\sum_{A} f_{A}(z) e_{A}$, where $f_{A}(z)$ are real valued. We suppose that $f$ is hypercomplex differentiable in $\Omega$ in the sense of [5], i.e. has a uniquely defined areolar derivative $f^{\prime}$ in each point of $\Omega$ (for the definition of an areolar derivative see [8]). Then $f^{\prime}$ can be expressed by $f^{\prime}=1 / 2\left(\partial_{0}-\partial_{\underline{x}}\right) f$, where $\partial_{0}:=\frac{\partial}{\partial x_{0}}, \quad \partial_{\underline{x}}:=$ $e_{1} \frac{\partial}{\partial x_{1}}+\cdots+e_{n} \frac{\partial}{\partial x_{n}}$. If now $D:=\partial_{0}+\partial_{\underline{x}}$ is the usual generalized Cauchy-Riemann differential operator, then, obviously $f^{\prime}=1 / 2 \bar{D} f$. Since in [5] it has been shown that a hypercomplex differentiable function satisfies the equation $D f=0$ ( $f$ is a monogenic function in the sense of Clifford Analysis), then it follows that in fact $f^{\prime}=\partial_{0} f$ like in the complex case. For our purpose we use also monogenic polynomials in terms of the hypercomplex monogenic variables $z_{k}=x_{k}-x_{0} e_{k}=-\frac{x e_{k}+e_{k} x}{2}, k=1,2, \cdots, n$, which leads to generalized powers of degree $m$ that are by convention symbolically written as $z_{1}^{\mu_{1}} \times \cdots z_{n}^{\mu_{n}}$. They are defined as an m-nary symmetric product by $z_{1}^{\mu_{1}} \times \cdots z_{n}^{\mu_{n}}=\frac{1}{m !} \sum_{\pi\left(i_{1}, \ldots, i_{m}\right)} z_{i_{1}} \cdots z_{i_{m}}$, where the sum is taken over all permutations of $\left(i_{1}, \ldots, i_{m}\right)$, (see [7] and [8]).

We will show the appearance of Special Function by a simple example related to a generalized exponential function. Therefore we ask for a Clifford algebra-valued function $E=E(x)$, defined and hypercomplex differentiable in $\mathbb{R}^{n+1}$ as solution of the initial value problem

$$
E^{\prime}=E, \quad E(0)=1 .
$$

Writing $x=x_{0}+\omega|\underline{x}|$ with $\omega=\frac{\underline{x}}{|x|}$ if $\underline{x} \neq 0$, we observe that

$$
\partial_{\underline{x}}|\underline{x}|=\omega \text { and } \partial_{\underline{x}} \omega=\frac{1-n}{|\underline{x}|} .
$$


As hypercomplex generalization of $e^{z}=e^{x_{0}}[\cos y+i \sin y]$ we ask for a monogenic function of the form

$$
E(x)=E\left(x_{0}+\underline{x}\right)=E\left(x_{0}+\omega|\underline{x}|\right)=e^{x_{0}}(F(|\underline{x}|)+\omega G(|\underline{x}|))
$$

with $F(|\underline{x}|)$ and $G(|\underline{x}|))$ as real valued functions of their arguments and $F(0)=1 ; G(0)=0$. Obviously, supposing that $E(x)$ is monogenic then $E(x)$ will be a solution of the initial value problem (1). The condition to be monogenic

$$
D E(x)=\left(\partial_{0}+\partial_{\underline{x}}\right)\left[e^{x_{0}}(F(|\underline{x}|)+\omega G(|\underline{x}|))\right]=0,
$$

can be reduced to the system of two ordinary differential equations

$$
\begin{gathered}
F^{\prime \prime}+\frac{n-1}{|\underline{x}|} F^{\prime}+F=0 . \\
G^{\prime \prime}+\frac{n-1}{|\underline{x}|} G^{\prime}+\left(1-\frac{n-1}{|\underline{x}|^{2}}\right) G=0 .
\end{gathered}
$$

by separation of the scalar- and vector part. But (2) and (3) are nothing else than differential equations for normalized Bessel functions of the first kind, namely

$$
\Gamma\left(\frac{n}{2}\right)\left(\frac{2}{|\underline{x}|}\right)^{\frac{n}{2}-1} J_{\frac{n}{2}-1}(|\underline{x}|) \quad \text { and } \Gamma\left(\frac{n}{2}\right)\left(\frac{2}{|\underline{x}|}\right)^{\frac{n}{2}-1} J_{\frac{n}{2}}(|\underline{x}|), \quad \text { resp. }
$$

The example shows the expression of the elementary hypercomplex function $E(x)$ by a pair of half-integral order Bessel functions in the form

$$
\operatorname{Exp}_{n}\left(x_{0}+\underline{x}\right)=e^{x_{0}} \Gamma\left(\frac{n}{2}\right)\left(\frac{2}{|\underline{x}|}\right)^{\frac{n}{2}-1}\left(J_{\frac{n}{2}-1}(|\underline{x}|)+\omega(x) J_{\frac{n}{2}}(|\underline{x}|)\right) .
$$

In this compact form we recognize easily the generalized hypercomplex counterparts of relations between the classical Bessel functions of first, second and third kind (Hankel functions) and their role as generalizations of the complex exponential, cosine and sine functions. The essential formal difference consists only in a change from the constant imaginary unit $i$ to the (variable) "hyperimaginary" unit $\omega$. Of course, in the same way, i.e. by simulating the complex structure of the elementary function in which we are interested in and applying the corresponding initial value problem we can obtain all their hypercomplex generalizations. For example, the hypercomplex hyperbolic cosine and hyperbolic sine are expressed by modified Bessel function and instead of the formal relationship with Hankel functions now naturally Macdonald functions come in. A more "exotic", but expected relationship can be shown between the hypercomplex counterpart of the inverse tangent and the LerchPhi-function. Conversely, we could say that some Special Functions could be treated versus elementary hypercomplex functions. Of course, this approach is not restricted to the exponential function and its relatives.

Going further to show some examples of results about intrinsic properties of the considered monogenic functions we pass to the study of homogeneous monogenic polynomials $\mathcal{P}_{k}^{n}(x)$ of degree $k$ which are generated by the generalized exponential generating function

$$
\operatorname{Exp}_{n}(x t)=e^{x_{0} t} \Gamma\left(\frac{n}{2}\right)\left(\frac{2}{|\underline{x}| t}\right)^{\frac{n}{2}-1}\left(J_{\frac{n}{2}-1}(|\underline{x}| t)+\omega(x) J_{\frac{n}{2}}(|\underline{x}| t)\right)=\sum_{k=0}^{\infty} \frac{\mathcal{P}_{k}^{n}(x) t^{k}}{k !} .
$$

Straightforward calculation shows that the polynomials can be obtained in terms of $x$ if $n \geq 1$ and $x, \bar{x}$ if $n \geq 2$, namely in the form

$$
\mathcal{P}_{k}^{n}(x)=\sum_{s=0}^{k} T_{s}^{k}(n) x^{k-s} \bar{x}^{s}, \quad \text { with } \quad T_{s}^{k}(n)=\frac{k !}{(n)_{k}} \frac{\left(\frac{n+1}{2}\right)_{(k-s)}\left(\frac{n-1}{2}\right)_{(s)}}{(k-s) ! s !},
$$

In [3] these $\mathcal{P}_{k}^{n}(x)$ have independently been studied from different point of views and it was proved directly that the sequence $\left(\mathcal{P}_{k}^{n}(x)\right)_{k>0}$ forms an Appell [1] sequence with respect to the hypercomplex derivative with the usual normalization $\mathcal{P}_{0}(x) \equiv 1$. Moreover, it holds $\mathcal{P}_{k}(1)=1$ and in the real and complex case the sequence is nothing else as the sequence of powers $x^{k}, \quad k=0,1, \ldots$ Of course, this is simply the consequence of 
being obtained by the ordinary real or complex exponential generating function. Notice that in the complex case, corresponding to $n=1$ with $e_{1}:=i$, for polynomials $\mathcal{P}_{k}(x)$ normalized by $\mathcal{P}_{k}(1)=1, k=0,1, \ldots$ follows immediately that $T_{0}^{k} \equiv 1$ and $T_{s}^{k} \equiv 0$, for $s>0$, since holomorphic functions in $\mathbb{C}$ have a series expansion which involves only the powers of $z=x_{0}+i x_{1}$ and not the conjugate variable $\bar{z}=x_{0}-i x_{1}$. In the hypercomplex case the $\mathcal{P}_{k}$ a priori may depend on $\bar{z}$.

Another interesting result can be obtained as consequence of their expression in terms of generalized powers. Using an elementary Cauchy-Kovalevskaya extension method for algebra-valued analytic functions of $\underline{x}$ (see [2]) and some argument of unique series representation it is easy to show that

$$
\mathcal{P}_{k}^{n}(x)=\mathbf{P}_{k}\left(z_{1}, \cdots, z_{n}\right)=c_{k}(n) \sum_{|\nu|=k} z_{1}^{\nu_{1}} \times \cdots \times z_{n}^{\nu_{n}}\left(\begin{array}{l}
k \\
\nu
\end{array}\right) e_{1}^{\nu_{1}} \times \cdots \times e_{n}^{\nu_{n}},
$$

where $\nu=\left(\nu_{1}, \cdots, \nu_{n}\right)$ is a multiindex and

$$
c_{k}(n):=\sum_{s=0}^{k}(-1)^{s} T_{s}^{k}(n)= \begin{cases}\frac{k ! !(n-2) ! !}{(n+k-1) ! !}, & \text { if } k \text { is odd } \\ c_{k-1}(n), & \text { if } k \text { is even }\end{cases}
$$

These different representations deserve now some special attention. Formula (6) together with the property that $\mathcal{P}_{k}(1)=\mathbf{P}_{k}\left(-e_{1},-e_{2}, \ldots,-e_{n}\right)=1$ surprisingly connects the uniquely defined real numbers $c_{k}$ with an expression which contains only linear combinations of the algebraic basic elements

$$
c_{k}(n)=\left[\sum_{|\nu|=k}(-1)^{k}\left(\begin{array}{l}
k \\
\nu
\end{array}\right)\left(e_{1}^{\nu_{1}} \times e_{2}^{\nu_{2}} \times \cdots \times e_{n}^{\nu_{n}}\right)^{2}\right]^{-1} .
$$

Moreover, for the simplest non-complex case $n=2$ those coefficients of the hypercomplex polynomial (6) are equal to

$$
c_{k}(2)=\frac{1}{2^{k}}\left(\begin{array}{c}
k \\
\left\lfloor\frac{k}{2}\right\rfloor
\end{array}\right)=\frac{\left(\begin{array}{c}
k \\
\left\lfloor\frac{k}{2}\right\rfloor
\end{array}\right)}{\sum_{s=0}^{k}\left(\begin{array}{l}
k \\
s
\end{array}\right)}, \text { where }\lfloor\rfloor \text { is the floor function, }
$$

which means that they are averaged (generalized) central binomial coefficients of the $k$-th line in the Pascal triangle. Since it holds for even $k$ that $c_{k-1}(2)=c_{k}(2)$, the sequence $\left(c_{k}(2)\right)_{k>0}$ is completely determined by the sequence $\left(c_{2 m}\right)_{m \geq 0}$ which involves only the ordinary central binomial coefficients $\left(\begin{array}{c}2 m \\ m\end{array}\right)$. It is worth noticing that $c_{2 m}=\frac{1}{2^{2 m}}\left(\begin{array}{c}2 m \\ m\end{array}\right)$ play an important role in combinatorics similar to that of the Catalan numbers $c_{2 m}=\frac{1}{m+1}\left(\begin{array}{c}2 m \\ m\end{array}\right)$.

Additional to this facts we can also establish a third expression of the $c_{k}(2)$. By using a binomial type expression of the considered polynomials in terms of $x_{0}$ and $|x|$ as well as its connection with the Bessel functions in its generating function (4), we can recognize that

$$
\begin{aligned}
\mathcal{P}_{k}\left(x_{0}+\underline{x}\right) & =\sum_{m=0}^{k} c_{m}(2)\left(\begin{array}{c}
k \\
m
\end{array}\right) x_{0}^{k-m}|\underline{x}|^{m}=\sum_{m=0}^{k} c_{m}(2)\left(\begin{array}{c}
k \\
m
\end{array}\right) x_{0}^{k-m}(\omega|\underline{x}|)^{m} \\
& =\sum_{m=0}^{k}\left(\begin{array}{c}
k \\
m
\end{array}\right) x_{0}^{k-m}|\underline{x}|^{m}\left(J_{0}^{(m)}(0)+\omega J_{1}^{(m)}(0)\right) .
\end{aligned}
$$

This means that

$$
J_{0}^{(m)}(0)+\omega J_{1}^{(m)}(0)=c_{m}(2) \omega^{m} .
$$

Therefore we can obtain an expression in terms of the values of the derivatives of the Bessel function $J_{0}$ in the origin. Relying on the well known property $J_{0}^{\prime}(z)=-J_{1}(z)$ and the values of $\omega^{m}$ it holds

$$
c_{2 k}(2)=(-1)^{k} J_{0}^{(2 k)}(0) \quad k=0,1, \ldots
$$


Finally we mention a binomial sum which involves the coefficients $c_{m}$ and can be deduced by a similar line of reasoning from aforementioned relations. It holds

$$
\sum_{m=0}^{k}(-1)^{m} c_{m}\left(\begin{array}{c}
2 k \\
2 m
\end{array}\right)=2^{k} L_{2 k}\left(\frac{1}{2} \sqrt{2}\right),
$$

where $L_{2 k}$ is the Legendre polynomial of degree $2 k$.

Here we could only have a glimpse of the basic ideas. In the talk we will explain them in more detail.

\section{Acknowledgments}

The research of the first author was partially supported by the Centro de Investigação e Desenvolvimento em Matemática e Aplicações (CIDMA) of the University of Aveiro, through the Portuguese Foundation for Science and Technology (FCT).

\section{References}

[1] P. Appell, Sur une class de polynomes, Ann. Sci. cole Norm. Sup. 9, 119-144 (1880).

[2] F. Brackx, R. Delanghe, and F. Sommen, Clifford Analysis, Pitman, Boston-London-Melbourne, 1982.

[3] M. I. Falcão, and H. R. Malonek, Generalized Exponentials through Appell sets in $\mathbb{R}^{n+1}$ and Bessel functions, AIP-Proceedings, Vol. 936, 738-741 (2007).

[4] R. Fueter, Functions of a Hyper Complex Variable, Lecture Notes written and supplemented by E. Bareiss, Fall Semester 1948/49, Univ. Zürich

[5] K. Gürlebeck, and H. Malonek, A hypercomplex derivative of monogenic functions in $\mathbb{R}^{n+1}$ and its applications Complex Variables Theory Appl. 39, 199-228 (1999).

[6] K. Gürlebeck, K. Habetha and W. Sprß ig, Holomorphic functions in the plane and n-dimensional space. Transl. from the German. (English) Basel: Birkhuser (2008)

[7] H. Malonek, A new hypercomplex structure of the euclidean space $\mathbb{R}^{m+1}$ and the concept of hypercomplex differentiability, Complex Variables 14, 25-33 (1990).

[8] H. Malonek, Selected topics in hypercomplex function theory, In: Clifford Algebras and Potential Theory, Eriksson, Sirkka-Liisa (ed.), University of Joensuu, Report Series 7 (2004), 111-150.

[9] H. R. Malonek, and M. I. Falcão, Clifford Analysis between Continuous and Discrete, AIP-Proceedings Proceedings, Vol. 1048, 682-685 (2008). 